\title{
Electrode for Force Sensor of Conductive Rubber
}

\author{
Masato Ohmukai $^{1}$, Yasushi Kami ${ }^{1}$, Ryosuke Matsuura ${ }^{2}$ \\ ${ }^{1}$ Department of Electrical and Computer Engineering, Akashi College of Technology, Hyogo, Japan \\ ${ }^{2}$ Department of Applied Analysis and Complex Dynamical Systems, Kyoto University, Kyoto, Japan \\ Email: ohmukai@akashi.ac.jp
}

Received July 2, 2012; revised August 3, 2012; accepted September 5, 2012

\begin{abstract}
Tactile sensors are believed to be a key element in order to realize robotic fingers to catch a fragile object without damage. Force sensitive conductive rubber is a low-cost material and then attractive for the application to tactile sensors. We have studied the effect of electrodes attached to the rubber sheets. We have tried four kinds of electrodes: vacuum deposited $\mathrm{Al}$, adhesive $\mathrm{Cu}$ tape, $\mathrm{Al}$ thin film sheet and silver paste. It can be concluded that vacuum deposited $\mathrm{Al}$ has the highest potential from the practical point of view; it has the widest dynamic range and good precision at the same time.
\end{abstract}

Keywords: Conductive Rubber; Force Sensor; Robot Finger; Electrode; Vacuum Deposition

\section{Introduction}

When robotic fingers pick an object with a feedback control, a force sensor plays a key role to control the actuation of the fingers [1]. If the picked object is a soft one, the robot needs a sophisticated software and hardware system at the same time to hold it gently because it requires a precise control of the force loaded to it. The force sensor should be the most elaborate part for the robotic fingers in a bid to realize humanoid fingers. The tactile information is used as a control parameter in dexterous manipulation of humanoid fingers [2-4].

The solutions of tactile sensors have been fully reported. The types of the sensors are in a wide variety. A kind of them is based on a strain gauge or piezo-resistive device. The resistance variation brought about by the applied strain in this kind of device. The sensor arrays of this type have been reported nowadays [5-7]. The arrays of micro sensors demonstrated the ability in position sensing. In some cases, micro-machine technique was included to form this kind of sophisticated sensors. Some researchers have studied three dimension sensor arrays that can detect shear forces [8]. Other sensors of different types have been reported so far: piezo-resistor $[9,10]$, capacitive [11,12], optical [13-15] and piezoelectric [1619] ones. Furthermore unique ideas for the sensors have been reported such as conducting fluid [20], organic transistors [21,22], electron tunneling [23,24], ultrasonic [25, 26], magneto-resistive [27,28] and field emission [29] applications. In order to miniature the sensing system, silicon-based micro-electromechanical system (MEMS) has been widely applied [30,31] to piezo-resistive [32-35] or capacitive [36,37] type of the tactile sensors.

We pay attention to a conductive rubber sheet that varies electric resistance with a force applied to it. The conductive rubber consists of an elastomer enriched with conductive filler particles. The resistance of the rubber sheet reduces when external compressive force is applied. The material shows isotropic conduction. The advantage of the rubber sheet as a force sensor of robotic fingers is the low cost of the flexible material as well as the large area sensing ability [38]. In order to deal successfully with the short of reliability of the material, it requires an improvement in the formation of this sensor with a help of an intelligent adaptive control at the same time. Although elaborate ideas for the rubber sensor have been reported, basic and important properties such as electrodes for the rubber have been left behind. This article then treats the study on the dependence of the electrodes to the output characteristics as a sensor.

We investigated several kinds of electrodes that sandwiched the rubber sheet from the front and the back sides in four styles: $\mathrm{Al}$ thin film, $\mathrm{Al}$ deposited on the rubber sheet by vacuum deposition method, $\mathrm{Cu}$ thin film with conductive adhesive tapes and silver paste spread on the surface of the conducting rubber. The relationship of the output voltage to applied force have been studied and discussed from the point of view of a force sensor in robotic finger. The selection of the electrode directly affects the performance of the sensor devices. Nevertheless the close investigations have not been performed so far. This is partly due to the insufficient reliability of the rubber. It is expected to make a prototype low-cost tactile sensor for robot fingers with the help of adequate feed- 
back control in the future.

\section{Experimental Details}

We used commercially available conductive rubber sheet (44 mm - $44 \mathrm{~mm}$ square and $15 \mu \mathrm{m}$ in thickness) in our experiments. We prepared four kinds of metal contacts to the rubber sheets as follows. Silver paste spread (P-255 by Nisshin EM Co. Ltd.) on the both surfaces of the rubber sheet and then dried naturally (sample A). The rubber sheet was sandwiched with two pieces of Al thin film sheet (12 $\mu \mathrm{m}$ in thickness) and then the perimeter was fixed with a piece of adhesive tape (sample B). Adhesive conductive tape was affixed to the both surfaces of the rubber (sample C). The adhesive conducting tape of CU35C (Sumitomo $3 \mathrm{M}$ Limited) consisted of $35 \mu \mathrm{m}$ thick copper foil and the electric conductive adhesive with metal particle dispersion. Sample D was formed by the deposition of Al by means of a vacuum evaporation method. In all cases, the metal electrodes covered about $90 \%$ of the surface on the both sides. The four kinds of electrodes were adopted from the point of view of simplicity in production. We show the photograph of sample $\mathrm{D}$ for example in Figure 1.

The prepared detecting circuit for the sensor is shown in Figure 2. The $\mathrm{r}$ denotes the resistance of the rubber sheet sensor that has several mega ohm without any force onto it and decreases down to the order of kilo ohm when the sensor is pressed by a finger. A resistor $(3.9 \mathrm{k} \Omega$ ) and a capacitor $(4.7 \mu \mathrm{F})$ connected together in parallel was connected in series to the sensor, as shown in Figure 2. A DC voltage of $5 \mathrm{~V}$ was applied to $\mathrm{V}$ in Figure 2. The output voltage Vo was taken across the added circuit elements. The capacitor worked to reduce high frequency noise.

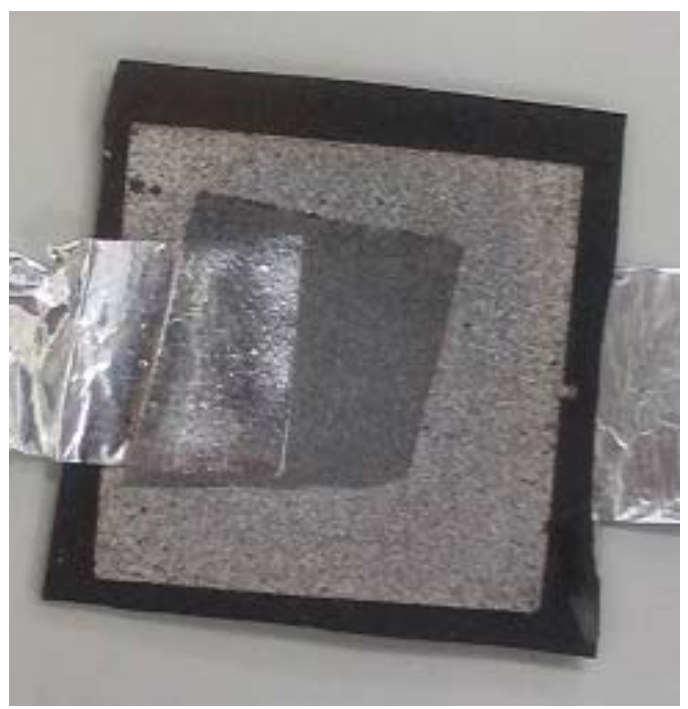

Figure 1. A photograph of sample D where Al was vacuum evaporated on the surface about $90 \%$ in area.
The Vo is transformed to a digital signal through an $\mathrm{A} / \mathrm{D}$ converter, and then directly acquired by a computer, followed by a signal process with widely used software such as Excel and Matlab.

In order to apply the force uniformly in the surface area, the sensor was put between two solid plates. Force was applied with the help of a hand compressor. The applied force was monitored simultaneously using a loadcell put together with the rubber sheet sensor in the hand compressor. The range of the applied force was under $200 \mathrm{~N}$ in our experiments. When the maximum value of $200 \mathrm{~N}$ is divided by the area of $44 \mathrm{~mm}$ square and then multiplied by the $3 \mathrm{~mm}$ square area, we obtain the value of $0.93 \mathrm{~N}$. This value well includes the typical force for the normal finger manipulation of 0.15 to $0.88 \mathrm{~N}$ (corresponding to 15 to $90 \mathrm{gf})[39,40]$.

\section{Results and Discussion}

We show here the four kinds of results in Figures 3-6, corresponding to samples $\mathrm{A}, \mathrm{B}, \mathrm{C}$ and $\mathrm{D}$, respectively,

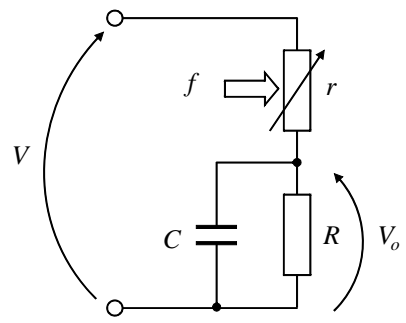

Figure 2. A detecting circuit for the rubber sensor $r$. Direct current voltage of $5 \mathrm{~V}$ was applied across the terminals ( $\mathrm{V}$ in the figure), and the voltage of Vo was measured.

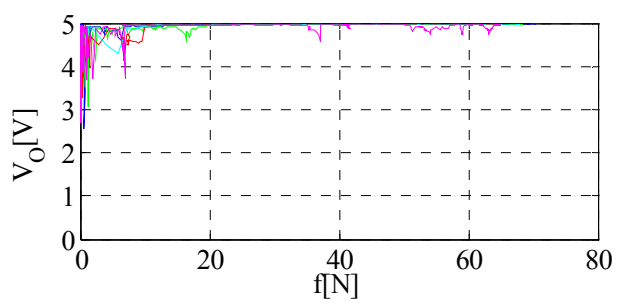

Figure 3. The dependence of Vo on the compressive force $f$ in sample A (measured 5 times): silver paste was spread on to the both surfaces of a rubber sheet.

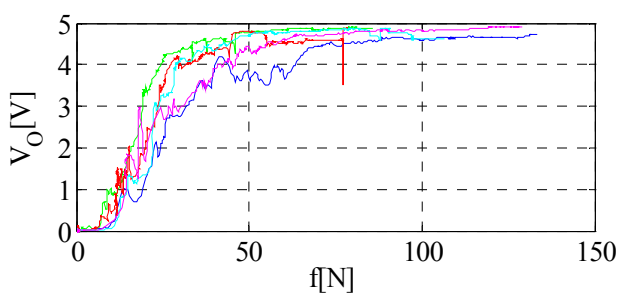

Figure 4. The dependence of Vo on the compressive force $f$ in sample B (measured 5 times): Al sheet was just put on to the both surfaces of a rubber sheet and only the perimeter was fixed with an adhesive tape. 


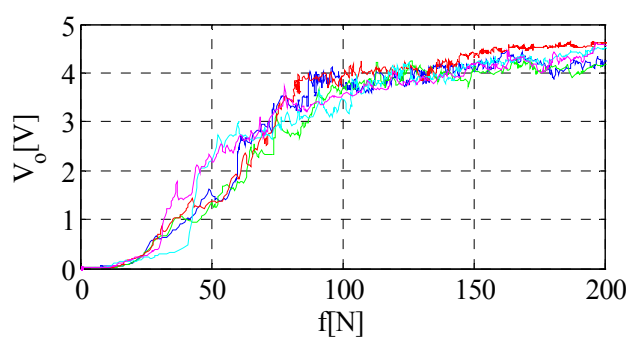

Figure 5. The dependence of Vo on the compressive force $f$ in sample $C$ (measured 5 times): a electrically conducting adhesive tape with copper foil was affixed to the both surfaces of a rubber sheet.

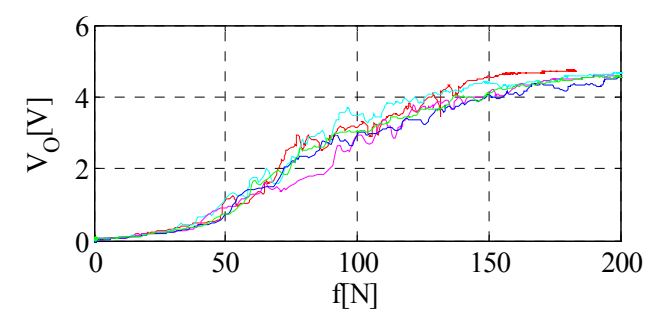

Figure 6. The dependence of Vo on the compressive force $f$ in sample D (measured 5 times): Al was deposited on the both surfaces by means of a vacuum evaporation method.

that is the relationship between output voltage Vo and applied force $\mathrm{f}$. We show Vo in the figures instead of the rubber resistance $r$, because we keep the feedback control in mind where the voltage Vo is of importance. Each figure includes several results to confirm the degree of reproducibility. Although the rubber sheets were well quality-controlled industrial products, the four kinds of results are interestingly quite different so that the difference is believed to be due to the effect of the electrode.

Figure 3 shows the fact that Vo was saturated no more than $10 \mathrm{~N}$. It is quite peculiar characteristics compared with the other three results that Vo increased gradually along the applied force. Silver paste forms aggregate of silver particles in a solvent. It is surely easy to be buried in concavities over the rubber rough surface when the paste was smeared. It is an advantage for the silver particles soak into the rubber concavities to obtain close contact to each other from the view of contact resistance reduction. When a compressive force is applied, silver particles possibly penetrate in the rubber from the both surfaces, to result in a shunt between the two electrodes. This might cause the saturation of Vo in a quite small region in the applied force. It stems from the move of silver particles inside the rubber.

We see the result of sample B next. The sensitivity is the biggest in the three cases excluding sample A; Vo saturated above $50 \mathrm{~N}$. The disadvantage of sample B is the worst reproducibility in the four. It possibly derived from the no fixation between the rubber and the electrodes. The compression force can cause the variation in the electrode contact state.
Figure 5 shows the Vo dependence on the applied force, when adhesive copper tape was adopted as electrodes (sample C). Saturation was observed above $80 \mathrm{~N}$ and so the dynamic range is twice as large as that of sample B. Though sensitivity is not large less than $25 \mathrm{~N}$ compared with that of sample B, it is noticeable that the reproducibility under $25 \mathrm{~N}$ is excellent. When the force was larger than $25 \mathrm{~N}$, Vo increased steeply as the force increased to $80 \mathrm{~N}$. In this region, however, the reproducibility was not good. We then define the two regions: stable region (under $25 \mathrm{~N}$ ) and unstable region (above 25 $\mathrm{N})$. The rubber sheet sensor is appropriate to be used in the stable region. In this sense, it can be said that samples $\mathrm{A}$ and $\mathrm{B}$ has no stable region. In the unstable region, the peeling off and sticking on between the rubber and adhesive tape irregularly occur and it seems to bear the dispersion of Vo, i.e. poor reproducibility.

Finally we see the case of sample D, where Al was deposited by evaporation, and discuss the characteristics here. The saturation of Vo was not observed in the range less than $200 \mathrm{~N}$; the dynamic range is in other words, largest in the four types. The two divided region was also observed in this sample where the stable region is stretched up to $40 \mathrm{~N}$. The stable region of sample D is largest in the four. It is surely because the Al electrodes and the rubber sheet is tightly bound to each other.

During the evaporation, $\mathrm{Al}$ gas reached the rubber surface even if the surface is quite rough. If the thickness of the deposited $\mathrm{Al}$ is smaller than the surface roughness, we can not obtain the continuous and crammed film and the electric conduction along the surface can not be well guaranteed. The thickness of the deposited Al films must be larger than the surface roughness of the rubber sheet. On the other hand, if the Al thickness is too large, it is presumed that the rubber becomes stiff consequently owing to the $\mathrm{Al}$ deposited layers. The optimum thickness of the $\mathrm{Al}$ electrodes is expected to be studied in the future.

The biggest problem of the conducting rubber sensor is generally the poor reproducibility derived from a inherent hysteresis property of the rubber. The problem can be possibly solved in part with the help of a histogram of frequency distribution of the sensor output and now under our study. At the same time, the electrodes strongly depend on the reproducibility as shown in this article. The improvement of the electrodes is actually of importance in order to develop the low-cost tactile sensor technology.

\section{Conclusions}

We have investigated four types of electrodes for a forcesensitive conducting rubber sensor. The electrical conducting rubber is expectedly applied to a tactile sensor with an advantage of low cost. The biggest problem of 
the material is to have hysteresis and poor reproducibility. The consideration of electrodes for the rubber sensor is inevitable but not yet well reported.

We found the output characteristics strongly depends on the form of electrodes, for example the dynamic range. The behavior of the output dependence should be divided into two regions: stable and unstable one. The stable region appears in a weaker force range and applicable to tactile sensors. In the unstable region, the rubber sensors show no good reproducibility. The range of the stable region is closely related to the contact state between the rubber surface and the electrode. As a result, the Al electrodes deposited by a vacuum evaporation method was found to have a largest stable region (0 to $40 \mathrm{~N})$. This stems from the tightly binding between the electrodes and the rubber sheet.

\section{REFERENCES}

[1] M. H. Lee and H. R. Nicholls, "Review Article Tactile Sensing for Mechatronics-A State of the Art Survey," Mechatronics, Vol. 9, No. 1, 1999, pp. 1-31. doi:10.1016/S0957-4158(98)00045-2

[2] Z. Li, P. Hsu and S. Sastry, "Grasping and Coordinated Manipulation by a Multifingered Robot Hand," International Journal of Robotics Research, Vol. 8, No. 4, 1989, pp. 33-50. doi:10.1177/027836498900800402

[3] A. D. Berger and P. K. Khosla, "Using Tactile Data for Real-Time Feedback," International Journal of Robotics Research, Vol. 10, No. 2, 1991, pp. 88-102. doi:10.1177/027836499101000202

[4] P. A. Schmidt, E. Mael and R. P. Wurtz, "A Sensor for Dynamic Tactile Information with Applications in $\mathrm{Hu}-$ man-Robot Interaction \& Object Exploration," Robotics and Autonomous Systems, Vol. 54, No. 12, 2006, pp. 1005-1014. doi:10.1016/j.robot.2006.05.013

[5] K. Kim, K. R. Lee, W. H. Kim, K. Park, T. Kim, J. Kim and J. J. Pak, "Polymer-Based Flexible Tactile Sensor Up to $32 \times 32$ Arrays Integrated with Interconnection Teminals," Sensors and Actuators A: Physical, Vol. 156, No. 2, 2009, pp. 284-291. doi:10.1016/j.sna.2009.08.015

[6] J. Engel, J. Chen and C. Liu, "Development of Polyimide Flexible Tactile Sensor Skin," Journal of Micromechanics and Microengineering, Vol. 13, No. 3, 2003, pp. 359366. doi:10.1088/0960-1317/13/3/302

[7] Y. Zhang, "Sensitivity Enhancement of a Micro-Scale Biomimetic Tactile Sensor with Epidermal Ridges," Journal of Micromechanics and Microengineering, Vol. 20, No. 8, 2010, Article ID: 085012. doi:10.1088/0960-1317/20/8/085012

[8] W.-C. Choi, "Polymer Micromachined Flexible Tactile Sensor for Three-Axial Toads Detection," Transactions on Electrical and Electronic Materials, Vol. 11, No. 3, 2010, pp. 130-133. doi:10.4313/TEEM.2010.11.3.130

[9] K. Noda, K. Hoshino, K. Matsumoto and I. Shimoyama, "A Shear Stress Sensor for Tactile Sensing with the Piezoresistive Cantilever Standing in Elastic Material," Sen- sors and Actuators A: Physical, Vol. 127, No. 2, 2006, pp. 295-301. doi:10.1016/j.sna.2005.09.023

[10] L. Beccai, S. Rocdella, L. Ascari, P. Valdastri, A. Sieber, M. Carrozza and P. Dario, "Development and Experimental Analysis of a Soft Compliant Tactile Microsensor for Anthropomorphic Artificial Hand," IEEE/ASME Transactions on Mechatronics, Vol. 13, No. 2, 2008, pp. 158168. doi:10.1109/TMECH.2008.918483

[11] H. Lee, J. Chung, S. Chang and E. Yoon, "Normal and Shear Force Measurement Using a Flexible Polymer Tactile Sensor with Embedded Multiple Capacitors," Journal of Microelectromechanical Systems, Vol. 17, No. 4, 2008, pp. 934-942. doi:10.1109/JMEMS.2008.921727

[12] S. Miyazaki and A. Ishida, "Capacitive Transducer for Continuous Measurement of Vertical Foot Force," Medical and Biological Engineering and Computing, Vol. 22, No. 4, 1984, pp. 309-316. doi:10.1007/BF02442098

[13] Y. Hasegawa, M. Shikida, D. Ogura, Y. Suzuki and K. Sato, "Fabrication of a Wearable Fabric Tactile Sensor Produced by Artificial Hollow Fiber," Journal of Micromechanics and Microengineering, Vol. 18, No. 8, 2008, Article ID: 085014. doi:10.1088/0960-1317/18/8/085014

[14] J.-S. Heo, J.-H. Chung and J.-J. Lee, "Tactile Sensor Arrays Using Fiber Bragg Grating," Sensors and Actuators A: Physical, Vol. 126, No. 2, 2006, pp. 312-327. doi:10.1016/j.sna.2005.10.048

[15] E. Cheung and V. L. Lumelsky, "A Sensitive Skin System for Motion Control of Robot Arm Manipulators," Robotics and Autonomous Systems, Vol. 10, No. 1, 1992, pp. 9-32. doi:10.1016/0921-8890(92)90012-N

[16] E. S. Kolesar, R. R. Reston, D. G. Ford and R. C. Fitch, "Multiplexed Piezoelectric Polymer Tactile Sensor," Journal of Robotic Systems, Vol. 9, No. 1, 1992, pp. 37-63. doi:10.1002/rob.4620090104

[17] J. Dargahi, M. Parameswaran and S. Payandeh, "A Micromachined Piezoelectric Tactile Sensor for an Endoscopic Grasper-Theory, Fabrication and Experiments," Journal of Microelectromechanical Systems, Vol. 9, No. 3, 2000, pp. 329-335. doi:10.1109/84.870059

[18] J. R. Flanagan and A. M. Wing, "Modulation of Grip Force with Load Force during Point-to-Point Arm Movements," Experimental Brain Research, Vol. 95, No. 1, 1993, pp. 131-143. doi:10.1007/BF00229662

[19] P. Dario and D. de Rossi, “Tactile Sensors and Gripping Challenge," IEEE Spectrum, Vol. 22, 1985, pp. 46-52.

[20] N. Wettels, V. Santos, R. Johansson and G. Loeb, "Biomimetric Tactile Sensor Array," Advanced Robotics, Vol. 22, No. 8, 2008, pp. 829-849. doi:10.1163/156855308X314533

[21] I. Manunza and A. Bonfiglio, "Pressure Sensing Using a Completely Flexible Organic Transistor," Biosensors and Bioelectronics, Vol. 22, No. 12, 2007, pp. 2775-2779. doi:10.1016/i.bios.2007.01.021

[22] T. Sekitani and T. Someya, "Stretchable, Large-Area Organic Electronics," Advanced Materials, Vol. 22, No. 2, 2010, pp. 2228-2246. doi:10.1002/adma.200904054

[23] D. Bloor, K. Donnelly, P. J. Hands, P. Laughlin and D. Lussey, "A Metal-Polymer Composite with Unusal Prop- 
erties," Journal of Physics D: Applied Physics, Vol. 38, No. 16, 2005, pp. 2851-2860. doi:10.1088/0022-3727/38/16/018

[24] V. Maheshwari and R. F. Saraf, "High-Resolution ThinFilm Device to Sense Texture by Touch," Science, Vol. 312, No. 5779, 2006, pp. 1501-1504. doi:10.1126/science. 1126216

[25] S. Ando and H. Shinoda, "Ultrasonic Emission Tactile Sensing," IEEE on Control System, Vol. 15, No. 1, 1995, pp. 61-69. doi:10.1109/37.341866

[26] R. S. Dahiya, M. Valle and L. Lorenzelli, "Spice Model of Lossy Piezoelectric Polymers," IEEE Transactions on Ultrasonics, Ferroelectrics and Frequency Control, Vol. 56, No. 2, 2009, pp. 387-396. doi:10.1109/TUFFC.2009.1048

[27] G. M. Krishna and K. Rajanna, "Tactile Sensor Based on Piezoelectric Resonance," IEEE Sensors Journal, Vol. 4, No. 5, 2004, pp. 691-697. doi:10.1109/JSEN.2004.833505

[28] T. J. Nelson, R. B. V. Dover, S. Jin, S. Hackwood and G. Beni, "Shear-Sensitive Magnetroresistive Robotic Tactile Sensor," IEEE Transactions on Magnetics, Vol. 22, No. 5, 1986, pp. 394-396. doi:10.1109/TMAG.1986.1064386

[29] Z. Wen, Y. Wu, Z. Zhang, S. Xu, S. Huang and Y. Li, "Development of an Integrated Vacuum Microelectronic Tactile Sensor Array," Sensors and Actuators A: Physical, Vol. 103, No. 3, 2003, pp. 301-306. doi:10.1016/S0924-4247(02)00392-8

[30] D. J. Beebe, A. S. Hsieh, D. D. Denton and R. G. Radwin, "A Silicon Force Sensor for Robotics and Medicine," Sensors and Actuators A: Physical, Vol. 50, No. 1-2, 1995, pp. 55-65. doi:10.1016/0924-4247(96)80085-9

[31] M. R. Wolffenbuttel and P. P. L. Regtien, "Polysilicon Bridges for the Realization of Tactile Sensors," Sensors and Actuators A: Physical, Vol. 26, No. 1-3, 1991, pp. 257-264. doi:10.1016/0924-4247(91)87002-K

[32] S. Sugiyama, K. Kawahata, M. Yoneda and I. Igarashi, "Tactile Image Detection Using a 1k-Element Silicon Pressure Sensor Array," Sensors and Actuators A: Physical, Vol. 22, No. 1-3, 1990, pp. 397-400.
[33] L. Liu, X. Zheng and L. Zhijian, "An Array Tactile Sensor with Piezoresistive Single-Crystal Silicon Diaphragm," Sensors and Actuators A: Physical, Vol. 35, No. 3, 1993, pp. 193-196. doi:10.1016/0924-4247(93)80151-6

[34] B. J. Kane, M. R. Cutkosky and G. T. A. Kovacs, “A Traction Stress Sensor Array for Use in High-Resolution Robotic Tactile Imaging," Journal of Microelectromechanical Systems, Vol. 9, No. 4, 2000, pp. 425-434. doi:10.1109/84.896763

[35] H. Takao, K. Sawada and M. Ishida, "Monolithic Silicon Smart Tactile Image Sensor with Integrated Strain Sensor Array on Pneumatically Swollen Single-Diaphragm Structure," IEEE Transactions on Electron Devices, Vol. 53, No. 5, 2006, pp. 1250-1259. doi:10.1109/TED.2006.872698

[36] Z. Chu, P. M. Saoor and S. Middelhoek, "Silicon ThreeAxial Tactile Sensor," Sensors and Actuators A: Physical, Vol. 54, No. 1-3, 1996, pp. 505-510. doi:10.1016/S0924-4247(95)01190-0

[37] M. Leineweber, G. Pelz, M. Schmidt, H. Kappert and G. Zimmer, "New Tactile Sensor Chip with Silicone Rubber Cover," Sensors and Actuators A: Physical, Vol. 84, No. 3, 2000, pp. 236-245. doi:10.1016/S0924-4247(00)00310-1

[38] Y.-J. Yang, M.-Y. Cheng, W.-Y. Chang, L.-C. Tsao, S.-A. Yang, W.-P. Shih, F.-Y. Chang, S.-H. Chang and K.-C. Fang, "An Integrated Flexible Temperature and Tactile Sensing Array Using PI-Copper Films," Sensors and Actuators A: Physical, Vol. 143, No. 1, 2008, pp. 143-153. doi:10.1016/j.sna.2007.10.077

[39] R. H. LaMotte and M. A. Srinivasan, "Tactile Discrimination of Shape: Responses of Slowly Adapting Mechanoreceptive Afferents to a Step Stroked across the Monkey Fingerpad," Journal of Neuroscience, Vol. 7, No. 6, 1987, pp. 1655-1671.

[40] R. H. LaMotte and M. A. Srinivasan, "Tactile Discrimination of Shape: Responses of Rapidly Adapting Mechanoreceptive Afferents to a Step Stroked across the Monkey Fingerpad," Journal of Neuroscience, Vol. 7, No. 6, 1987, pp. 1672-1681. 